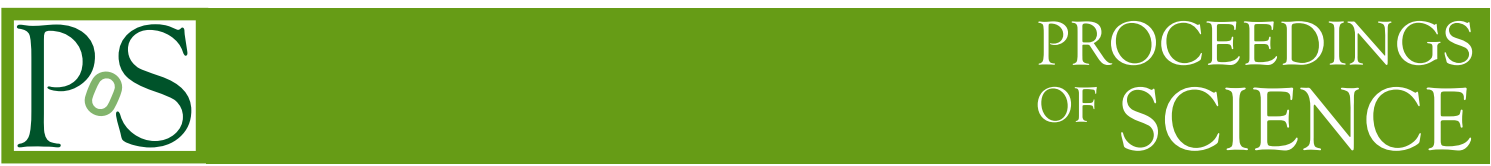

\title{
Early new physics searches with leptons at LHC
}

\section{Claudio Gatti ${ }^{* \dagger}$}

Laboratori Nazionali di Frascati - INFN, Via E. Fermi 40, 00044 Frascati (Rome) Italy

E-mail: claudio.gatti@lnf.infn.it

Several models predict the existence of new gauge bosons leading to final states with high $p_{T}$ leptons: heavier copies of Standard Model $W$ and $Z$, decaying to dilepton or lepton with missing $E_{T}$; bosons coupling to right-handed fermions, as in Left-Right symmetric models, where the decay of a heavy neutrino leads to final states with leptons and jets. The prospect for early discoveries, with integrated luminosity of about $100 \mathrm{pb}^{-1}$, using ATLAS and CMS detectors, are discussed.

European Physical Society Europhysics Conference on High Energy Physics July 16-22, 2009

Krakow, Poland

\footnotetext{
*Speaker.

${ }^{\dagger}$ On behalf of the ATLAS and CMS Collaborations.
} 


\section{Introduction}

Several models predict the existence of new gauge bosons with a width to mass ratio of about $1 \%$, decaying to lepton pairs [1]. In the sequential standard model (SSM), $Z^{\prime}$ and $W^{\prime}$ have the same couplings of the $Z$ and $W$ in the Standard Model (SM), but have higher masses. At hadron colliders, new bosons may be produced in the $s$ channel from quark anti-quark annihilation. $Z^{\prime}$ bosons may show up as narrow resonances in the invariant-mass distribution of dileptons, while $W^{\prime}$ may decay to $l v$ and be identified by the high-energy charged lepton and large missing transverse energy $\left(E_{T}^{\text {miss }}\right)$. Left-Right symmetric models predict the existence of a $W^{\prime}$ coupling to right-handed fermions ( $W_{R}$ in the following). In this case, a heavy right-handed neutrino may decay leading to a different final state with no missing energy.

Experiments at the Tevatron collider exclude the existence of such bosons with masses below $1 \mathrm{TeV}$ [2-8]. An improvement of these results is limited by the energy available in the $p \bar{p}$ center of mass (CM) system. At LHC, with a CM energy of $14 \mathrm{TeV}$, the production cross section for bosons with mass of about $1 \mathrm{TeV}$, ranges from a fraction to few $\mathrm{pb}$, allowing us to probe this mass region already with an integrated luminosity of about $100 \mathrm{pb}^{-1}$.

\section{2. $Z^{\prime} \rightarrow e e$}

The dielectron channel is very promising because of the high resolution of the electromagnetic calorimeters, dominated at these energies by the constant term. This term is below $1 \%$ for both ATLAS [9] and CMS [10]. Moreover, the irreducible background, due to Drell-Yan (DY) process, is negligible above $1 \mathrm{TeV}$ with respect to the expected signal. On the other hand, dijet events with transverse momentum above $300 \mathrm{GeV}$, have a cross section more than 4 orders of magnitude higher than our signal, and a very good electron-jet rejection is needed. This is achieved by identifying electrons on the basis of ratio of hadronic and electromagnetic energy; track association; $E / p$; shower shape. Residual QCD background can be estimated from data control samples. Identification efficiency is studied using $Z \rightarrow e e$ events. A precision of about $1 \%$ can be achieved with $100 \mathrm{pb}^{-1}$ for several regions in the $p_{T}-\eta$ space up to $100 \mathrm{GeV}$. Monte Carlo simulation is needed for the extrapolation to higher energies. The energy resolution could be degraded by a bad knowledge of the material in front of the calorimeter and by a non uniform calibration of the electromagnetic calorimeter. A detailed study of these effects will be done using photon conversions in the inner detector material, $E / p$ distribution of inclusive electrons, and finally exploiting the mass constraint in $Z \rightarrow e e$ decays. This will allow us to keep the constant term below $1 \%$ with an integrated luminosity of $100 \mathrm{pb}^{-1}$.

The discovery potential has been studied by both CMS [12] and ATLAS [11] Collaborations, for several models and masses of the new boson. Both the studies show that the discovery of an SSM $Z^{\prime}$ with $1 \mathrm{TeV}$ mass, is already possible with $10 \mathrm{pb}^{-1}$ at $14 \mathrm{TeV}$ as shown in figure 1. A more recent study [13] shows that with a center-of-mass energy of $10 \mathrm{TeV}$ twice the integrated luminosity is required for the same discovery.

\section{3. $Z^{\prime} \rightarrow \mu \mu$}

Contrary to the dielectron case, in the dimuon channel the reconstructed resonance width is 

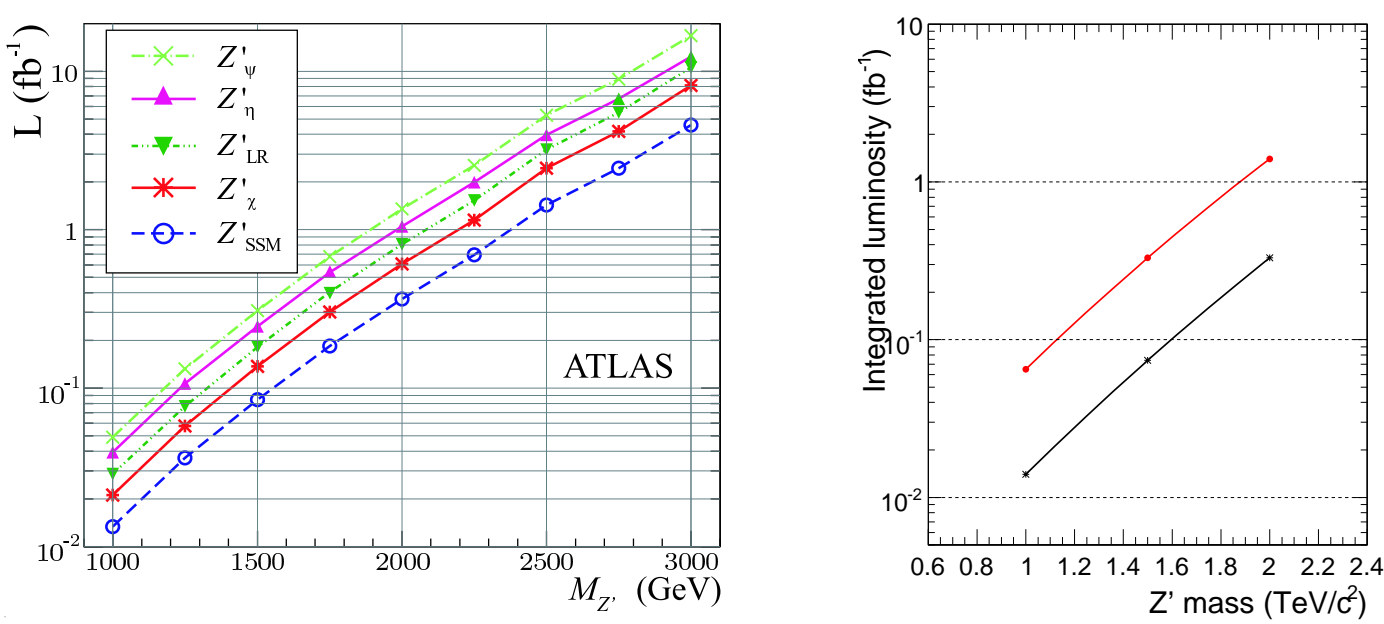

Figure 1: Integrated luminosity needed for a $5 \sigma$ discovery of $Z^{\prime} \rightarrow l l$ as a function of the $Z^{\prime}$ mass. Left: ATLAS results for the dielectron channel and for several $Z^{\prime}$ models. Right: CMS results for the dimuon channel for SSM (bottom) and for $E_{6}$ grand-unification (top) models.

dominated by the momentum resolution. At high momenta, the intrinsic resolution of the detector is the dominant contribution and the alignment of the tracking system becomes crucial. For instance, to achieve the nominal resolution of about $6 \%$ at $500 \mathrm{GeV}$, ATLAS needs an alignment better than $30 \mu \mathrm{m}$ in the muon spectrometer and $10 \mu \mathrm{m}$ in the silicon modules of the inner detector. This accuracy can be achieved by using optical and track-based alignment, as shown by tests performed on cosmic ray data. Reconstruction efficiency, momentum scale and resolution can be measured with $Z \rightarrow \mu \mu$ events with accuracy better than $1 \%$ with $100 \mathrm{pb}^{-1}$ of integrated luminosity.

The luminosity required for a $5 \sigma$ discovery is similar to that found for the dielectron case, as shown in the right panel of figure 1 [11] [14]. Both experiments have tested the effect of misalignments in the detector, reconstructing the events with a non ideal knowledge of the geometry. For instance, introducing random misalignments between muon chambers in the ATLAS spectrometer, with a very conservative value of the rms of about $300 \mu \mathrm{m}$, the luminosity required to discover a SSM $Z^{\prime}$ with $1 \mathrm{TeV}$ mass, increases only by about $30 \%$.

4. $W^{\prime} \rightarrow l v$

$W^{\prime} \rightarrow l v$ events are selected by looking for a high momentum charged lepton and large $E_{T}^{m i s s}$. The main background is due to the continuum process $p p \rightarrow W^{*} \rightarrow l v, t \bar{t}$ events and high- $p_{T}$ jets. In particular, large $E_{T}^{\text {miss }}$ may be reconstructed when high- $p_{T}$ jets point toward cracks or gaps in the calorimeters, or close to regions with instrumental problems. Therefore, a deep understanding of the reconstructed $E_{T}^{\text {miss }}$ is needed. Several performance studies will be performed using minimum bias events and $Z$ and $W$ leptonic decays.

The CMS discovery potential for $W^{\prime} \rightarrow e v$ as a function of the mass is shown in figure 2 (Left). Less than $10 \mathrm{pb}^{-1}$ are needed for the discovery of a $1 \mathrm{TeV}$ boson. Similar results have been obtained by ATLAS. For the muon channel, ATLAS shows that about a factor 2 higher luminosity is needed due to larger instrumental uncertainties on the muon reconstruction. 

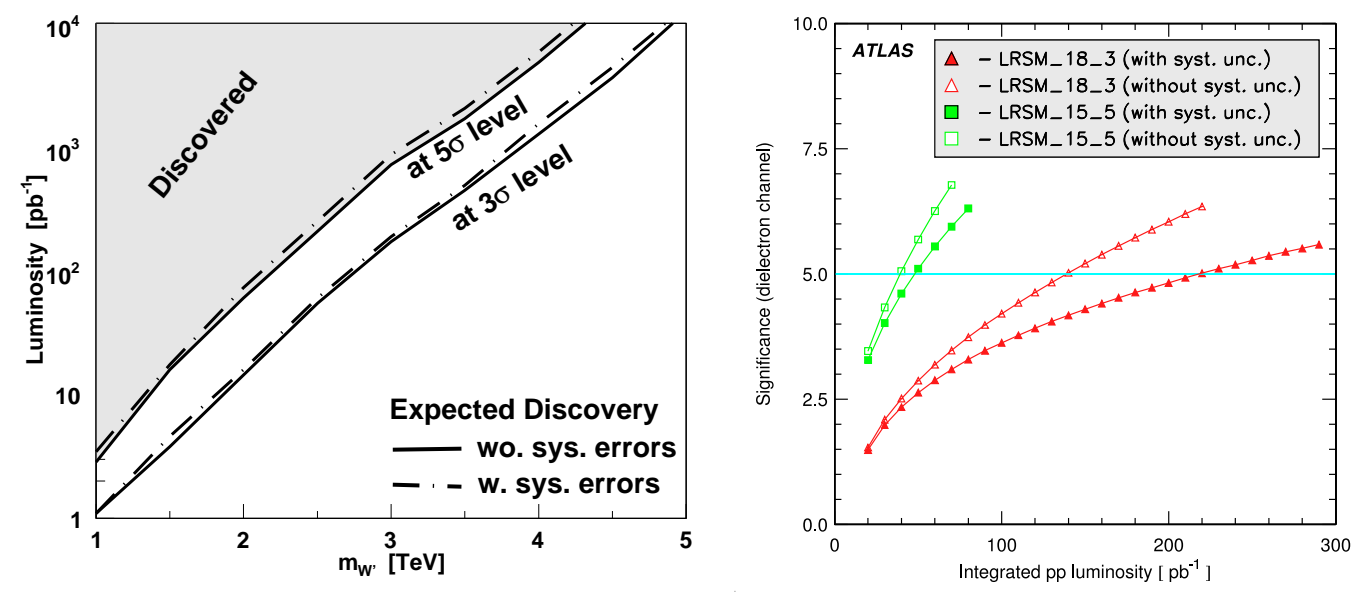

Figure 2: Left: CMS discovery potential for $W^{\prime} \rightarrow e v$ as a function of the mass. Right: Signal significance in ATLAS as a function of the integrated luminosity for a $W_{R}$ boson in the electron channel, for the two scenarios discussed in the text.

5. $W_{R} \rightarrow l l j j$

Left-Right symmetric models predict the production of a $W_{R}$ boson decaying into lepton and right-handed Majorana neutrino $(N)$. The neutrino may decay to a lepton and two quarks, leading to a final state with two leptons and two jets. ATLAS have studied the discovery potential for both the muon and electron channels, in two possible mass scenarios [11]: $M_{W}=1800 \mathrm{GeV}$ and $M_{N}=300$ $\mathrm{GeV} ; M_{W}=1500 \mathrm{GeV}$ and $M_{N}=500 \mathrm{GeV}$. Events are selected requiring high- $p_{T}$ leptons and jets, with large dilepton invariant mass and total transverse energy. The main background is due to DY, $t \bar{t}$ and boson pair production.

The signal significance for the electron channel is shown in figure 2 (Right), for the two mass scenarios. About $40 \mathrm{pb}^{-1}$ are needed for the discovery of a $W_{R}$ of $1500 \mathrm{GeV}$ mass. Similar results have been obtained for the muon channel.

\section{Conclusion}

The large CM energy available at LHC will allow us search for new bosons with masses up to few $\mathrm{TeV}$. With an integrated luminosity of $100 \mathrm{pb}^{-1}$ we could test several models for boson masses up to $2 \mathrm{TeV}$. However, since lower $\mathrm{CM}$ energy implies lower production cross sections, a higher integrated luminosity will be needed for a machine operating with lower beam energy. At $10 \mathrm{TeV}$ CM energy, about a factor two higher integrated luminosity is needed, and a factor about 4 is needed at $8 \mathrm{TeV}$.

The possible discovery of a new boson with early data is made more difficult by the non optimal alignment and calibration of the detector. Several studies will be performed as the first data will come using several control samples: minimum bias events; inclusive muons and electrons; $\mathrm{Z}$ and $\mathrm{W}$ decays. These samples will also be used to measure reconstruction efficiencies. An integrated luminosity of $100 \mathrm{pb}^{-1}$ will be enough to reach a detector performance close to the 
design value and a knowledge of the efficiencies at \% level or better. The integrated luminosity required for these studies is limited by the production of weak bosons, and it increases almost linearly as the machine energy is decreased. However, with a lower performance of the detectors, similar to what is expected during the first days of data taking, the required luminosity is increased at most by about $30 \%$. This has been tested by including misalignments in the detector geometry, or by checking the effect of a lower energy resolution.

Another uncertainty comes from the expected background yield and shape. Several methods have been implemented to determine these quantities directly from data control samples. With 100 $\mathrm{pb}^{-1}$ we could estimate several backgrounds at 10 or $20 \%$ level. Finally, other systematic errors arising from the knowledge of the PDF or from QED and EW corrections have been considered and included in the final error. In conclusion, ATLAS and CMS will be able to discover new bosons at the $\mathrm{TeV}$ scale, or improve the limits set by the Tevatron experiments, already with the first 100 $\mathrm{pb}^{-1}$ collected at LHC, even with lower performance and lower CM energy.

\section{References}

[1] Reviews on $W^{\prime}$ and $Z^{\prime}$ in C. Amsler et al. (Particle Data Group), Physics Letters B667, 1 (2008).

[2] V.M. Abazov et al. [D0 Collaboration], Phys. Rev. Lett. 100211803 (2008)

[3] V.M. Abazov et al. [D0 Collaboration], Phys. Rev. Lett. 100031804 (2008)

[4] The D0 Collaboration, Conference Note 5923-CONF (2009).

[5] The D0 Collaboration, Conference Note 4577-CONF (2004).

[6] A. Abulencia et al., The CDF Collaboration, Phys. Rev. D 75, 091101 (2007).

[7] T. Aaltonen et al., The CDF Collaboration, Phys. Rev. Lett. 99, 171802 (2007).

[8] A. Abulencia et al., The CDF Collaboration, Phys. Rev. Lett. 95, 252001 (2005).

[9] The ATLAS Collaboration, JINST $3 \mathbf{S 0 8 0 0 3}$

[10] The CMS Collaboration, JINST $3 \mathbf{S 0 8 0 0 4}$

[11] The ATLAS Collaboration, CERN-OPEN-2008-020

[12] The CMS Collaboration, CMS PAS EXO-08-001

[13] The CMS Collaboration, CMS PAS EXO-09-006

[14] The CMS Collaboration, CMS PAS SBM-07-002

[15] The CMS Collaboration, CMS PAS EXO-08-004 